\title{
Effect of laser therapy on plasma expression of VEGF and bFGF in infants with cutaneous hemangioma
}

\author{
FAGANG WANG, RONGJIAN XU, QIAN XU, YONGQIAN CAO, LI LIN and WEI DANG \\ Department of Aesthetic Plastic and Burn Surgery, Provincial Hospital Affiliated to Shandong University, \\ Jinan, Shandong 250021, P.R. China
}

Received June 9, 2015; Accepted November 1, 2016

DOI: $10.3892 / \mathrm{ol} .2017 .5640$

\begin{abstract}
The present study aimed to investigate the effect of laser therapy on the expression of vascular endothelial growth factor (VEGF) and basic fibroblast growth factor (bFGF) in the plasma of infants diagnosed with cutaneous hemangioma, in order to identify biomarkers for assessing the clinical efficacy of laser therapy. In total, 109 infants with superficial abdominal hemangioma received laser treatment, of which 74 were diagnosed in the proliferation phase, 20 in the regression phase and 15 in the involution phase. In addition, 10 infants without cutaneous hemangioma were recruited as normal controls. The concentrations of VEGF and bFGF in peripheral plasma samples were measured using ELISAs. Dynamic changes in the VEGF and bFGF concentrations of 23 infants diagnosed in the proliferation phase were compared before and after laser therapy. The plasma concentration of VEGF in the proliferation phase group was significantly higher compared with that in the regression phase, involution phase and normal control groups (all $\mathrm{P}<0.01$ ), whereas it did not differ significantly among the regression phase, involution phase and normal control groups (all $\mathrm{P}>0.05$ ). The plasma concentration of bFGF in the proliferation phase group was significantly higher compared with that in the regression phase, involution phase and normal control groups (all $\mathrm{P}<0.01$ ), while the bFGF level did not differ significantly among the regression phase, involution phase and normal control groups (all $\mathrm{P}>0.05$ ). Following laser therapy, the plasma concentrations of VEGF and bFGF in infants with cutaneous hemangioma were significantly decreased (both $\mathrm{P}<0.01)$. These results suggested that VEGF and bFGF may serve as reliable indexes to evaluate the clinical efficacy of laser therapy for treating infantile cutaneous hemangioma.
\end{abstract}

Correspondence to: Dr Fagang Wang, Department of Aesthetic Plastic and Burn Surgery, Provincial Hospital Affiliated to Shandong University, 324 Jingwu Road, Jinan, Shandong 250021, P.R. China E-mail: fagangwang2015@sina.com

Key words: infants, hemangioma, laser therapy, plasma, vascular endothelial growth factor, basic fibroblast growth factor

\section{Introduction}

Hemangioma is a common type of infantile congenital benign tumor, which is characterized by abnormal proliferation of vascular tissues. It usually appears in the first weeks of life and grows most rapidly over the initial 6 months. Typically, growth is complete and involution has commenced by 12 months. Hemangioma proceeds through three phases including proliferation, regression and involution. If not properly controlled, it is likely to cause significant cosmetic injury or even threaten the patient's life (1-3). The cause of hemangioma is currently unknown. However, previous studies have suggested that the expression levels of vascular endothelial growth factor (VEGF) and basic fibroblast growth factor (bFGF) are closely correlated with the proliferation of the endothelial cells of a hemangioma (4-9). Takahashi et al (10) demonstrated that VEGF was highly expressed in the endothelial cells of proliferative phase hemangioma, whereas it was undetectable during the regression phase, indicating that VEGF is closely associated with the incidence and involution of hemangioma. Bielenbery et al (11) reported that high levels of bFGF were expressed in proliferative phase hemangioma tissue, whereas normal expression levels of bFGF were detected on the surface of hemangioma in the regression phase. Currently, laser therapy, hormonal therapy, radiotherapy, interferon treatment and surgery are used to treat hemangioma. Laser therapy has been increasingly adopted to manage infantile cutaneous hemangioma because it is a noninvasive technique that yields no secondary scarring or pigment alteration (12). The clinical efficacy of laser therapy for treating hemangioma is predominantly evaluated by a medical history and physical examination. However, a simple and reliable detection method is urgently required.

In the present study, the plasma levels of VEGF and bFGF, which are related to vascular hyperplasia, were detected in patients with hemangioma in varying phases to investigate the effect of laser therapy on plasma VEGF and bFGF concentrations in infants diagnosed with cutaneous hemangioma. The aim of this study was to identify simple and objective biomarkers for evaluating the clinical efficacy of laser therapy.

\section{Patients and methods}

Patient enrollment. A total of 109 patients, including 44 males and 65 females, diagnosed with superficial abdominal 
hemangioma at the Department of Burn and Plastic Surgery of Shandong Provincial Hospital Affiliated to Shandong University (Jinan, China) between October 2009 and August 2012 were recruited in the present study. Written informed consent was obtained from all patients, and ethical approval was obtained from Shandong Provincial Hospital Affiliated to Shandong University (Jinan, China). The inclusion criteria were as follows: i) Those with complete clinical data; ii) those who were able to comply with the doctors' prescription and complete the entire treatment; and iii) those who were able to complete the subsequent follow-up. Of the 109 cases, 74 were assigned to the proliferation phase group, 20 to the regression phase group and 15 to the involution phase group. Furthermore, 10 patients (5 male) without hemangioma were enrolled in the control group, and 23 patients with hemangioma who were aged 40 days to 6 months, with lesion areas ranging from 4-30 $\mathrm{cm}^{2}$, were randomly selected to observe the superficial abdominal hemangioma and assigned to the dynamic observation group (Table I). All patients were admitted to our hospital for the first time and had not previously received laser therapy.

Patients with hemangioma were staged according to previously described criteria (6). Briefly, hemangiomas were defined as in the proliferation phase if they rapidly grew upon admission and were observed as having a bright coloration and hard texture (Fig. 1A). Hemangiomas were classified as in the regression phase if their growth was stable or gradually diminished, and if they became white and the tumor texture was softened (Fig. 1B). Hemangiomas were defined as in the involution phase if they were completely stable and no further treatment was required (Fig. 1C).

Blood sample collection. Venous blood (2 ml) was collected from all patients in sterile anticoagulation tubes and centrifuged at 3,500 x $g$ for $10 \mathrm{~min}$ at $4^{\circ} \mathrm{C}$. Subsequently, the plasma was collected, distributed into sterile EP tubes and stored at $-80^{\circ} \mathrm{C}$ until use.

Measurement of VEGF and bFGF concentrations. The plasma levels of VEGF and bFGF were determined using ELISAs (Human VEGF ELISA kit; Abcam, Cambridge, MA, USA).

Laser therapy. Prior to laser treatment, the site, size, onset time and severity of the hemangioma were evaluated. The lesional skin was anesthetized using 2\% lignocaine (Teva Pharmaceuticals, Petah Tikva, Israel). Laser therapy was performed by a proficient physician using an N-Lite Pulsed Dye Laser (ICN Photonics Ltd., Llanelli, UK) at a wavelength of $585 \mathrm{~nm}$, pulse length of $0.45 \mathrm{~ms}$, spot diameter of $5 \mathrm{~mm}$, at an energy density of $7.5 \mathrm{~J} / \mathrm{cm}^{2}$ and a frequency of $1.5 \mathrm{~Hz}$. Following 3 cycles of laser treatment, the tumor site was disinfected with benzalkonium bromide at a concentration of $0.1 \mathrm{mg} / \mathrm{ml}$ (Sigma-Aldrich; EMD Millipore, Billerica, MA, USA) and intermittently cooled using an ice bag for 20-30 min. Subsequently, mupirocin ointment (Teva Pharmaceuticals) was applied three times per day for 4 days. Each cycle of laser therapy was delivered at a time interval of 1 month until the end of the treatment.

Statistical analysis. SPSS 16.0 software (SPSS, Inc., Chicago, IL, USA) was used for statistical analyses. Differences among multiple groups were analyzed using one-way analysis of variance (ANOVA). Differences between two groups were analyzed by Least Significance Difference test. $\mathrm{P}<0.05$ was considered to indicate a statistically significant difference.

\section{Results}

Changes in the plasma levels of VEGF and bFGF in patients with different phases of hemangioma. The plasma concentrations of VEGF in the different groups are shown in Table II. ANOVA revealed that the plasma concentration of VEGF was significantly different among the proliferation phase, regression phase, involution phase and control groups $(F=27.824$; $\mathrm{P}<0.001)$. LSD demonstrated that the plasma level of VEGF in the proliferation phase group significantly differed from those in the regression phase, involution phase and control groups (all $\mathrm{P}=0<0.001$ ). Furthermore, it was demonstrated that there was no significant difference in the plasma concentration of VEGF between any two groups among the regression phase, involution phase and control groups (all $\mathrm{P}>0.05$ ).

The plasma concentrations of bFGF in different patients are shown in Table III. ANOVA revealed that the plasma concentration of bFGF significantly differed among the proliferation phase, regression phase, involution phase and control groups $(F=38.738 ; \mathrm{P}<0.001)$. LSD demonstrated that the plasma level of bFGF in the proliferation phase group significantly differed from those in the regression phase, involution phase and control groups (all $\mathrm{P}<0.001$ ). In addition, it was shown that there was no significant difference in the plasma concentration of bFGF between any two groups among the regression phase, involution phase and control groups (all $\mathrm{P}>0.05$ ).

Changes in the plasma levels of VEGF and bFGF in the dynamic observation group before and after laser therapy. The plasma concentrations of VEGF and bFGF in the dynamic observation group before and after laser therapy at the same time points are shown in Table IV and Fig. 2. Statistical analysis revealed that the plasma concentrations of VEGF and bFGF in the dynamic observation group at the same time points differed significantly prior to and following laser therapy (VEGF: $t=7.478, \mathrm{P}=0.000$; bFGF: $t=7.785, \mathrm{P}<0.001$ ).

\section{Discussion}

In 1982, Mulliken and Glowacki (6) proposed a biological classification of vascular birthmarks on the basis of clinical manifestations, histopathological features and natural history, which was subsequently adopted by The International Society for the Study of Vascular Anomalies in 1996 (7). Since then, congenital vascular diseases have been further classified into hemangiomas and vascular abnormalities (8). Hemangioma, which results from abnormal proliferation of vascular endothelial cells, is one of the most common types of congenital benign tumors during infancy, and rapidly grows and proliferates prior to the infant reaching 1 year-of-age (9). Previous studies have demonstrated that VEGF, bFGF and their receptors are closely associated with the proliferation of infantile cutaneous hemangioma (10). Takahashi et al detected upregulated expression of recombinant VEGF-16539 in transgenic rabbit models, and a histological examination revealed hemangioma in the rabbit liver. Immunohistochemical analysis demonstrated that human 
Table I. Demographic data of all participants.

\begin{tabular}{lccccc}
\hline Parameters & $\begin{array}{c}\text { Proliferation phase } \\
\text { group }(\mathrm{n}=74)\end{array}$ & $\begin{array}{c}\text { Regression } \\
\text { phase }(\mathrm{n}=20)\end{array}$ & $\begin{array}{c}\text { Involution phase } \\
\text { group }(\mathrm{n}=15)\end{array}$ & $\begin{array}{c}\text { Control group } \\
(\mathrm{n}=10)\end{array}$ & $\begin{array}{c}\text { Dynamic observation } \\
\text { group }(\mathrm{n}=23)\end{array}$ \\
\hline Age (months) & $1.0-9.0$ & $4.0-12.0$ & $11.5-26.0$ & $1.3-24.0$ & $1.3-6.0$ \\
Male/female & $27 / 47$ & $8 / 12$ & $9 / 6$ & $5 / 5$ & $8 / 15$ \\
Lesion size $\left(\mathrm{cm}^{2}\right)$ & $3-36$ & $3-20$ & 0 & 0 & $4-30$ \\
\hline
\end{tabular}

Table II. Comparison of the plasma concentration of VEGF among the different groups (means \pm standard deviation).

\begin{tabular}{lcccc}
\hline Parameters & $\begin{array}{c}\text { Proliferation phase } \\
\text { group }(\mathrm{n}=45)\end{array}$ & $\begin{array}{c}\text { Regression phase } \\
\text { group }(\mathrm{n}=18)\end{array}$ & $\begin{array}{c}\text { Involution phase } \\
\text { group }(\mathrm{n}=11)\end{array}$ & $\begin{array}{c}\text { Control group } \\
(\mathrm{n}=10)\end{array}$ \\
\hline Age (months) & $3.88 \pm 2.16$ & $9.17 \pm 2.68$ & $19.18 \pm 5.80$ & $7.83 \pm 6.32$ \\
Male/female & $16 / 29$ & $7 / 11$ & $7 / 4$ & $5 / 5$ \\
Body weight $(\mathrm{kg})$ & $7.05 \pm 1.54$ & $9.92 \pm 2.87$ & $11.32 \pm 1.66$ & 0 \\
Lesion size $\left(\mathrm{cm}^{2}\right)$ & $6.67 \pm 6.94$ & $7.44 \pm 11.52$ & $05 \pm 2.05$ \\
VEGF $\left(\mathrm{pg} / \mathrm{ml}^{2}\right.$ & $268.64 \pm 256.16^{\mathrm{a}}$ & $42.89 \pm 37.90$ & $25.55 \pm 21.76$ & $26.50 \pm 23.50$ \\
\hline
\end{tabular}

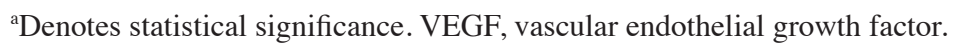
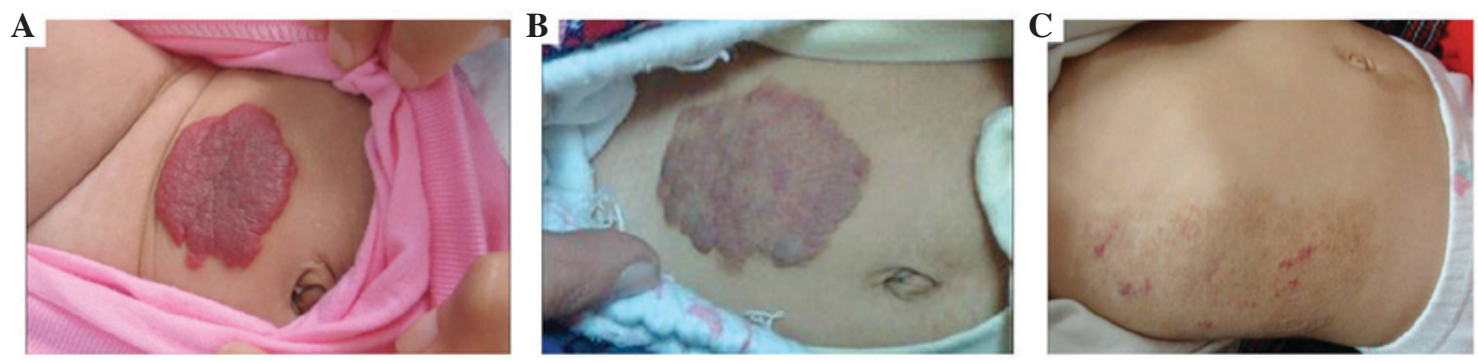

Figure 1. Photographs revealing different phases of infantile hemangioma. (A) Proliferation phase, (B) regression phase and (C) involution phase.

VEGF-165 was expressed in hepatocytes rather than in rabbit plasma, suggesting that upregulated expression of VEGF-165 results in the incidence of hemangioma in transgenic rabbit models (13). Takahashi et al (10) analyzed hemangioma clinical specimens using immunohistochemical staining and found that the expression of VEGF was significantly upregulated in proliferative phase hemangioma, whereas it was markedly downregulated in hemangiomas in the regression and involution phases. Bielenberg et al (11) utilized in situ hybridization and immunohistochemical analysis to measure the expression of VEGF and bFGF in hemangioma samples at various phases, and demonstrated that both VEGF and bFGF were highly expressed, suggesting that VEGF and bFGF are closely correlated with the incidence of hemangioma.

The majority of hemangiomas disappear by themselves and do not require any treatment. However, hemangiomas should be treated if they affect important structures, such as the eyes, nose, ears or windpipe, if they keep growing at a high speed, and if they become ulcerated and painful. Radiotherapy, hormonal therapy, interferon treatment, surgery and laser therapy are commonly employed for the treatment of hemangiomas (12). In plastic surgery, laser therapy has been frequently applied to treat superficial vascular illnesses including infantile cutaneous hemangioma and portwine stains (14). No complications, including hyperplastic or atrophic scars, have been reported after laser therapy. Over the past few decades, laser therapy has been widely applied to the treatment of hemangiomas at our hospital, resulting in the accumulation of clinical experience and clinical data. Furthermore, a favorable clinical efficacy and high degree of satisfaction have been obtained. In the present study, 109 infants diagnosed with hemangioma and undergoing laser therapy at our institution between October 2009 and August 2012 were enrolled, including 74 patients with hemangioma in the proliferation phase, 20 in the regression phase and 15 in the involution phase. The potential effect of laser therapy on the plasma concentrations of VEGF and bFGF in infants was evaluated to explore a simple and reliable parameter to assess the clinical efficacy of laser therapy in the management of hemangioma.

The exact pathogenesis of hemangioma remains largely unknown. However, several studies have suggested the importance of angiogenic factors in the incidence and proliferation of hemangioma, which is likely caused by a disrupted balance in the stimulating and inhibitory factors of angiogenesis (15-17). 
Table III. Comparison of the plasma concentrations of bFGF among the different groups (means \pm standard deviation).

\begin{tabular}{|c|c|c|c|c|}
\hline Parameters & $\begin{array}{l}\text { Proliferation phase } \\
\quad \text { group }(n=51)\end{array}$ & $\begin{array}{l}\text { Regression phase } \\
\text { group }(n=20)\end{array}$ & $\begin{array}{l}\text { Involution phase } \\
\text { group }(n=15)\end{array}$ & $\begin{array}{l}\text { Control group } \\
\qquad(\mathrm{n}=10)\end{array}$ \\
\hline Age (months) & $3.84 \pm 2.06$ & $9.20 \pm 2.54$ & $18.15 \pm 5.86$ & $7.83 \pm 6.32$ \\
\hline Male/female & $19 / 32$ & $8 / 12$ & $9 / 6$ & $5 / 5$ \\
\hline Body weight (kg) & $6.95 \pm 1.52$ & $10.20 \pm 3.04$ & $11.23 \pm 1.69$ & $8.05 \pm 2.05$ \\
\hline Lesion size $\left(\mathrm{cm}^{2}\right)$ & $6.37 \pm 6.60$ & $6.90 \pm 11.03$ & 0 & 0 \\
\hline bFGF (pg/ml) & $490.75 \pm 255.21^{\mathrm{a}}$ & $129.10 \pm 130.41$ & $12.93 \pm 6.55$ & $7.40 \pm 4.88$ \\
\hline
\end{tabular}

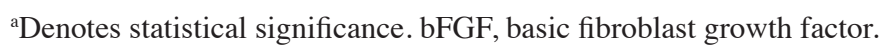

Table IV. Comparison of plasma levels of VEGF and bFGF in the dynamic observation group before and after laser therapy (means \pm standard deviation).

Parameters

Age (months)

Male/female

Body weight $(\mathrm{kg})$

Lesion size $\left(\mathrm{cm}^{2}\right)$

VEGF (pg/ml)

bFGF (pg/ml)
Before laser therapy

After laser therapy

$$
\begin{gathered}
3.79 \pm 1.86 \\
8 / 15 \\
7.67 \pm 2.39 \\
9.46 \pm 10.99 \\
63.22 \pm 38.84^{\mathrm{a}} \\
433.83 \pm 207.71^{\mathrm{a}}
\end{gathered}
$$

$$
\begin{gathered}
6.31 \pm 1.76 \\
8 / 15 \\
9.13 \pm 2.39 \\
9.46 \pm 10.99 \\
30.74 \pm 31.22 \\
195.09 \pm 152.62
\end{gathered}
$$

${ }^{a}$ Denotes statistical significance (VEGF: $t=7.478, \mathrm{P}<0.000$; bFGF: $t=7.785, \mathrm{P}<0.000$ ). VEGF, vascular endothelial growth factor; bFGF, basic fibroblast growth factor.
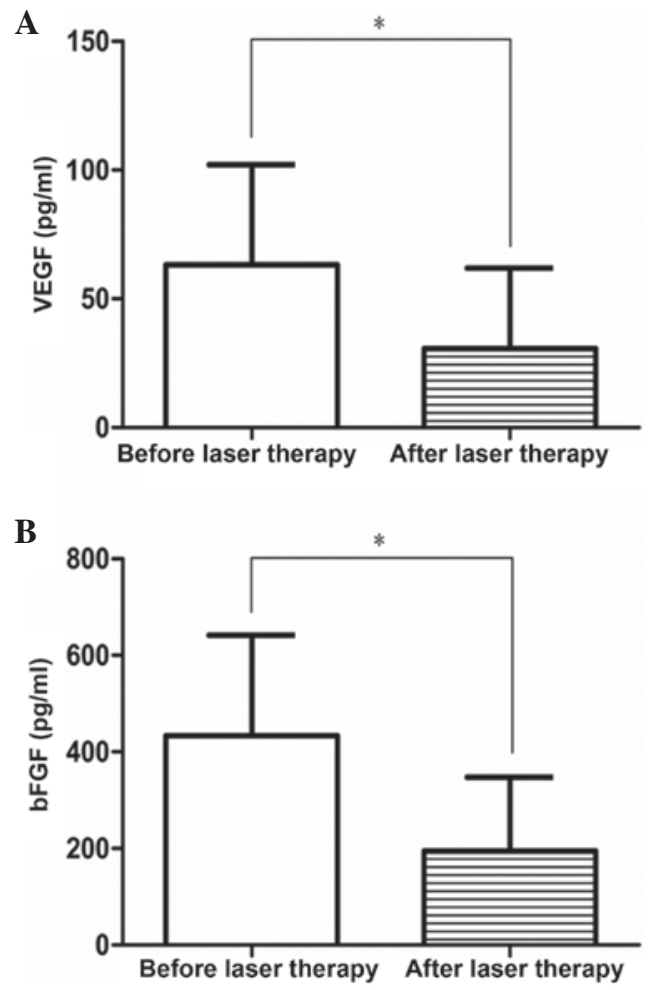

Figure 2. Changes in the plasma levels of VEGF and bFGF in the dynamic observation group before and after laser therapy. (A) Changes in the plasma concentration of VEGF before and after laser therapy $(\mathrm{P}<0.001)$. (B) Changes in the plasma concentration of bFGF before and after laser therapy $(\mathrm{P}<0.001)$. VEGF, vascular endothelial growth factor; bFGF, basic fibroblast growth factor. ${ }^{*} \mathrm{P}<0.05$.
There is evidence that the expression levels of VEGF and bFGF in the peripheral blood of infants with proliferative phase hemangioma were significantly upregulated compared with those in patients with hemangiomas at the regression phase and normal controls, whereas they did not significantly differ between regression phase infants and healthy counterparts (16). In the present study, the plasma concentrations of VEGF and bFGF in the proliferation phase group were significantly higher compared with those in the regression phase, involution phase and control groups, while no significant difference was observed among the regression phase, involution phase and control groups. After several cycles of laser therapy, the plasma concentrations of VEGF and bFGF were declined to within the normal ranges $(<40$ and $<300 \mathrm{pg} /$ $\mathrm{ml}$, respectively), from the proliferation phase to the regression phase. For each hemangioma patient, the plasma levels of VEGF and bFGF were significantly reduced after laser therapy, suggesting that the laser therapy was able to lower the plasma concentrations of VEGF and bFGF.

To date, the majority of studies have focused on the histopathological evaluation of hemangioma specimens of different phases, employing quantitative polymerase chain reaction and immunohistochemical staining $(17,18)$. Conversely, the present study collected peripheral blood samples from infants diagnosed with hemangioma, which was a safe, noninvasive, sensitive and convenient method for assessing the different phases, and may be widely applied in clinical practice following validation by subsequent large-scale clinical trials. Furthermore, the present study observed that individuals exhibited 
different levels of sensitivity to laser therapy. The majority of the infants were highly sensitive to laser therapy; the plasma concentrations of VEGF and bFGF were rapidly decreased following laser therapy and favorable clinical outcomes were obtained. However, a minority of infants exhibited low sensitivity to laser treatment; the plasma concentrations of VEGF and bFGF were maintained at relatively high levels following laser therapy. Therefore, the combined use of laser treatment with hormonal therapy or injection of medicines, such as propranolol, vincristine and interferon, may be considered (16).

In the present study, the plasma concentrations of VEGF and bFGF rapidly declined following laser therapy. Although the exact mechanism is unknown, it may result from the high-energy laser selectively promoting the thermal coagulation of hemoglobin, eventually leading to vascular occlusion. Hemoglobin absorbs the laser energy, forms thrombi and causes injuries to endothelial cells (18), which is directly caused by laser therapy or indirectly by vascular occlusion. The complications of thrombosis and vascular occlusion after laser therapy block topical blood circulation to the hemangioma and lead to hypoxia, thereby causing the swelling, degeneration and necrosis of vascular endothelial cells. Upon the incidence of vascular endothelial cell injury, the expression levels of VEGF and bFGF were decreased and the proliferation of hemangioma was reduced accordingly. Therefore, the authors of the present study hypothesized that laser therapy may cause endothelial cell injury and subsequently affect the expression of VEGF and bFGF in endothelial cells, thereby promoting the progression of the proliferation phase into the regression phase. However, the underlying mechanism requires further elucidation. Furthermore, due to the difficulty in collecting and preserving tumor samples, the sample size was relatively small. Therefore, subsequent studies with a larger sample size should be performed.

In conclusion, the present study demonstrated that, after several cycles of laser therapy, the cutaneous hemangiomas of infants with proliferative phase hemangioma were alleviated to the regression phase. Simultaneously, the high plasma concentrations of VEGF and bFGF in the proliferation phase were reduced to within their normal ranges during the regression phase, which was consistent with the clinical characteristics. Therefore, the plasma levels of VEGF and bFGF may be used to evaluate the clinical efficacy of laser therapy.

\section{Acknowledgements}

This study was supported by grants from the Natural Science Foundation of Shandong Province (funding no.ZR2013HM058).

\section{References}

1. Zhao FY, Gao Y, Wu MJ, Luo QF, Liu Y and Xu ZQ: Diagnosis and therapy on hemangiomas and vascular malformation in view of the new classification. Beijing Da Xue Xue Bao 41: 21-27, 2009 (In Chinese).

2. Smolinski KN and Yan AC: Hemangiomas of infancy: Clinical and biological characteristics. Clin Pediatr (Phila) 44: 747-766, 2005.

3. Jackson IT: Hemangiomas. Eur J Plast Surg 31: 275-280, 2008.

4. Hohenleutner U, Landthaler M, Hamm H and Sebastian G: Hemangiomas of infancy and childhood. J Dtsch Dermatol Ges 5: 334-338, 2007 (In English, German).

5. Huang QL and Feng XL: Advances of research on infantile hemangioma associated factors. Zhongguo Meirong Yixue 20: 880-882, 2011 (In Chinese).

6. Mulliken JB and Glowacki J: Hemangiomas and vascular malformations in infants and children: A classification based on endothelial characteristics. Plast Reconstr Surg 69: 412-422, 1982.

7. Hu QH, Lin XX and Wang W: Association between VEGF and its receptors and proliferative hemangiomas. Zhong Hua Zheng Xing Wai Ke Za Zhi 18: 237, 2002 (In Chinese).

8. Su T, Zhao YF and Zhang WF: Proliferative hemangioma and bFGF. Guo Wai Yi Xue 32: 113-114, 2005 (In Chinese).

9. Przewratil P, Sitkiewicz A and Andrzejewska E: Local serum levels of vascular endothelial growth factor in infantile hemangioma: Intriguing mechanism of endothelial growth. Cytokine 42: 141-147, 2010.

10. Takahashi K, Mulliken JB, Kozakewich HP, Rogers RA, Folkman J and Ezekowitz RA: Cellular markers that distinguish the phases of hemangioma during infancy and childhood. J Clin Invest 93: 2357-2364, 1994.

11. Bielenberg DR, Bucana CD, Sanchez R, Mulliken JB, Folkman J and Fidler IJ: Progressive growth of infantile cutaneous hemangiomas is directly correlated with hyperplasia and angiogenesis of adjacent epidermis and inversely correlated with expression of the endogenous angiogenesis inhibitor, IFN-beta. Int J Oncol 14: 401-408, 1999.

12. Huang Y, Wang JF and Lv LQ: The development of laser treatment for infant hemangioma. Zhong Guo Yi Xue Wen Zhai-Pi Fu Ke Xue 27: 349-351, 2010.

13. Arndt S, Poser I, Schubert T, Moser M and Bosserhoff AK: Cloning and functional characterization of a new Ski homolog, Fussel-18, specifically expressed in neuronal tissues. Lab Invest 85: 1330-1341, 2005.

14. Ohmori S and Huang CK: Recent progress in the treatment of portwine staining by argon laser: Some observations on the prognostic value of relative spectro-reflectance (RSR) and the histological classification of the lesions. Br J Plast Surg 34: 249-257, 1981.

15. North PE, Waner M, Mizeracki A and Mihm MC Jr: GLUT1: A newly discovered immunohistochemical marker for juvenile hemangiomas. Hum Pathology 31: 11-22, 2000.

16. Chang J, Most D, Bresnick S, Mehrara B, Steinbrech DS, Reinisch J, Longaker MT and Turk AE: Proliferative hemangiomas: Analysis of cytokine gene expression and angiogenesis. Plast Reconstr Surg 103: 1-9; discussion 10, 1999.

17. Folkman J: Seminars in medicine of the Beth Israel Hospital, Boston. Clinical applications of research on angiogenesis. N Engl J Med 333: 1757-1763, 1995.

18. Ritter MR, Butschek RA, Friedlander M and Friedlander SF: Pathogenesis of infantile haemangioma: New molecular and cellular insights. Expert Rev Mol Med 9: 1-19, 2007. 